\title{
INTEGRALLY CLOSED AND COMPLETE ORDERED QUASIGROUPS AND LOOPS
}

\author{
PHILLIP A. HARTMAN ${ }^{1}$
}

\begin{abstract}
We generalize the well-known results on embedding a partially ordered group in its Dedekind extension by showing that, with the appropriate definition of integral closure, any partially ordered quasigroup (loop) $G$ can be embedded in a complete partially ordered quasigroup (loop) if and on!y if $G$ is integrally closed. If $G$ is directed as well, then its Dedekind extension is a complete lattice-ordered quasigroup (loop). Furthermore, any complete fully ordered quasigroup (loop) has, with one exception, the real numbers with their usual ordering as its underlying set. The quasigroup (loop) operation, however, need not be ordinary addition as it is in the group case. On the other hand, a complete, strongly power associative fully ordered loop is either the integers or the real numbers with ordinary addition.
\end{abstract}

1. Introduction. Following the notation of [5] we will write quasigroups and loops additively. A partially ordered quasigroup is a quasigroup $G$ with a partial order satisfying conditions (i) and (ii) of [5]. If the order is a lattice-order (full order) then $G$ is called a lattice-ordered (fully ordered) quasigroup. It is easily shown that $G$ satisfies conditions (iii)(viii) of [5]. The reader may refer to [2], [3] and [5] for terminology and basic results not given here.

2. Integral closure and completeness. Traditionally, a partially ordered group is said to be integrally closed provided $n a \leqq b(n=1,2, \cdots)$ implies $a \leqq 0$ (see [2, p. 290]). Iseki [8] extended this definition to fully ordered loops by letting $n a$ denote the $n$th right multiple of $a$. He, however, used the term "right Archimedean" which we will do here so that we may reserve the term "integrally closed" for another use with quasigroups. The following was first proved for fully ordered loops [8].

THEOREM 1. If $L$ is a complete lattice-ordered loop then $L$ is right Archimedean. 1971.

Presented to the Society, November 20, 1971; received by the editors September 30,

AMS 1970 subject classifications. Primary 06A50; Secondary 06A55, 20 N05.

Key words and phrases. Integral closure, complete, right Archimedean, Dedekind completion, strong power associativity.

1 This paper is part of the author's doctoral dissertation written under the direction of Professor Trevor Evans at Emory University. 
Proof. Suppose $L$ is a complete lattice-ordered loop with $a, b \in L$ and $n a \leqq b(n=1,2, \cdots)$. Then the set $\{n a \mid n=1,2, \cdots\}$ is bounded above by $b$ and hence has a least upper bound $c$. Thus $c+a=(\bigvee\{n a \mid n=1,2, \cdots\})+$ $a=\bigvee\{n a \mid n=2,3, \cdots\} \leqq c$, so that $a \leqq 0$. Hence $L$ is right Archimedean.

Although an integraily closed lattice-ordered group can be embedded in a complete lattice-ordered group (see [6, pp. 92-95]), it is not apparent that a right Archimedean lattice-ordered loop can be embedded in a complete lattice-ordered loop. Furthermore, the definition "right Archimedean" is useless for quasigroups since in general they have no neutral element. We now formulate a definition of "integral closure" for partially ordered quasigroups (loops) that will guarantee that the Dedekind completion of a directed quasigroup (loop) is also a quasigroup (loop).

Definition. Let $G$ be a partially ordered quasigroup. $G$ is said to be integrally closed provided that for any sequence $\left\{u_{n}\right\}$ in $G$ that is bounded below and for any $a, b \in G$, if any of the following conditions are satisfied then $\left\{u_{n}\right\}$ is increasing (or equivalently $a \geqq b$ ):

(i) $-u_{n}+b=-u_{n+1}+a(n=1,2, \cdots)$,

(ii) $b-u_{n}=a-u_{n+1}(n=1,2, \cdots)$,

(iii) $u_{n+1}+b=u_{n}+a(n=1,2, \cdots)$,

(iv) $b+u_{n+1}=a+u_{n}(n=1,2, \cdots)$.

If $G$ is associative, that is a group, this definition is equivalent to the usual definition of "integral closure" for groups, and in fact conditions (i)-(iv) are equivalent to each other. However it does not appear that if $G$ is a loop, the presence of a neutral element would permit a less complicated definition.

THEOREM 2. If $G$ is a complete lattice-ordered quasigroup then $G$ is integrally closed. Conversely, an integrally closed directed quasigroup can be embedded in a complete lattice-ordered quasigroup.

Proof. Suppose that $G$ is a complete lattice-ordered quasigroup. We will show that if $\left\{u_{n}\right\}$ is a sequence in $G$ that is bounded below and if $a$ and $b$ are elements of $G$ such that (i) of the definition is satisfied then $a \geqq b$. The proofs involving conditions (ii)-(iv) are similar. Since $G$ is complete $\left\{u_{n}\right\}$ has a greatest lower bound $c$. Suppose (i) is satisfied, so that $u_{n+1}=a-\left(-u_{n}+b\right) \quad(n=1,2, \cdots)$. Since $c=\bigwedge\left\{u_{n} \mid n=1,2, \cdots\right\}$ we have

$$
\begin{aligned}
a-(-c+b) & =a-\left(-\bigwedge\left\{u_{n} \mid n=1,2, \cdots\right\}+b\right) \\
& =\bigwedge\left\{a-\left(-u_{n}+b\right) \mid n=1,2, \cdots\right\} \\
& =\bigwedge\left\{u_{n} \mid n=1,2, \cdots\right\} \geqq c .
\end{aligned}
$$

Thus we have $a-(-c+b) \geqq c$ so that $a \geqq c+(-c+b)=b$. 
Conversely suppose that $G$ is an integrally closed directed quasigroup. We will construct the Dedekind extension of $G$. For each subset $A$ of $G$ let $U(A)$ denote the set of all upper bounds of $A$, let $L(A)$ denote the set of all lower bounds of $A$, and let $A^{\#}$ denote $L(U(A))$. Let $G^{\#}=\left\{A^{\#} \mid A\right.$ is a nonempty subset of $G$ that is bounded above $\}$. We note that if $A \in G^{\#}$ then $A$ is bounded above and $A^{\#}=A$.

A binary operation $\oplus$ may be defined on $G^{\#}$ by letting $A \oplus B=(A+B)^{\#}$ for all $A, B \in G^{\#}$. Exactly as in the case where $G$ is a group (see [6, pp. 94-95]) we may prove the following:

LemMA 3. (i) $G^{\#}$ is a conditionally complete lattice with respect to inclusion $(\subset)$,

(ii) the map $a \rightarrow\{a\}^{\#}$ embeds $G$ in $G^{\#}$ with preservation of order, least upper bounds, greatest lower bounds, and addition,

(iii) if $A, B, C \in G^{\#}$ then $C \ominus(A \vee B)=(C \oplus A) \vee(C \oplus B)$ and $(A \vee B) \oplus C=$ $(A \oplus C) \vee(B \oplus C)$,

(iv) if $a \in G$ and $B$ is a nonempty subset of $G$ then $a+B^{\#}=(a+B)^{\#}$,

(v) if $A \in G^{\#}$ and $B$ is a nonempty subset of $G$ that is bounded above then $\left(A+B^{\#}\right)^{\#}=(A+B)^{\#}$ and $\left(B^{\#}+A\right)^{\#}=(B+A)^{\#}$.

We define $\ominus A \oplus B=(-U(A)+B)^{\#}$ and $B \ominus A=(B-U(A))^{\#}$ for each $A, B \in G^{\#}$. To show that $G^{\#}$ is a quasigroup with respect to the three operations $A \odot B, \ominus A \ominus B$ and $B \ominus A$ it suffices to show that the following identities are satisfied:

(i) $A \oplus(\ominus A \oplus B)=B$,

(ii) $(B \ni A) \ominus A=B$,

(iii) $\ominus A \oplus(A \odot B)=B$,

(iv) $(B \oplus A) \ominus A=B$.

These are obtained respectively from conditions (i)-(iv) of the definition of integral closure. We will demonstrate (i) and (iii).

Let $A, B \in G^{\#}$. If $x \in U(B), a \in A, u \in U(A)$ and $b \in B$ then $x \geqq b=$ $a+(-a+b) \geqq a+(-u+b)$, so that $U(B) \subseteq U\left(A+\left(-U^{\prime}(A)+B\right)\right)$. On the other hand, suppose $x \in U(A+(-U(A)+B))$. If $b \in B, a \in A$ and $u \in U(A)$ then $x \geqq a+(-u+b)$, so that $x-(-u+b) \geqq a$. Thus $x-(-U(A)+B) \subseteq$ $U(A)$. We set $u_{1}=u$ and $u_{n+1}=x-\left(-u_{n}+b\right)(n=1,2, \cdots)$. The sequence $\left\{u_{n}\right\}$ is bounded below by $a$, and so by (i) of the definition of integral closure we have $x \geqq b$. Hence $x \in U(B)$ and $U(A+(-U(A)+B)) \subseteq U(B)$. Thus $U^{\prime}(A+(-U(A)+B))=U(B)$ and the identity (i) follows immediately.

Now we show that the identity (iii) is satisfied. Let $A, B \in G^{\#}$. If $x \in U(B)$, $u \in U(A), a \in A$ and $b \in B$, then $x \geqq b=-a+(a+b) \geqq-u+(a+b)$, so that $x \in U(-U(A)+(A+B)$ ). Hence $U(B) \subseteq U(-U(A)+(A+B))$. On the other hand, suppose $x \in U(-U(A)+(A+B))$. If $b \in B, a \in A$, and $u \in U(A)$ then 
$x \geqq-u+(a+b)$, so that $(u+x)-b \geqq a$. We set $u_{1}=u$ and $u_{n+1}=\left(u_{n}+x\right)-b$ $(n=1,2, \cdots)$. The sequence $\left\{u_{n}\right\}$ is bounded below by $a$, and so by (iii) of the definition of integral closure we have $x \geqq b$. Hence $x \in U(B)$ and $U(-U(A)+(A+B)) \subseteq U(B)$. Thus $U(-U(A)+(A+B))=U(B)$. From Lemma 3(iv) we have $u+(-u+(A+B))^{\#}=(A+B)^{\#}$, so that

$$
(-u+(A+B))^{\#}=-u+(A+B)^{\#}
$$

for each $u \in U(A)$. Hence we have

$$
\begin{aligned}
L(U(B)) & =L\left(U(-U(A)+(A+B)) \subseteq\left(-U(A)+(A+B)^{\#}\right)^{\#}\right. \\
& =\left(\bigcup\left\{-u+(A+B)^{\#} \mid u \in U(A)\right\}\right)^{\#} \\
& =\left(\bigcup\left\{(-u+(A+B))^{\#} \mid u \in U(A)\right\}\right)^{\#} \\
& \subseteq\left(-U(A)+(A+B)^{\#}\right),
\end{aligned}
$$

so that $B=B^{\#}=\left(-U(A)+(A+B)^{\#}\right)^{\#}=\ominus A \subseteq(A \subseteq B)$.

Thus we conclude that $G^{\#}$ is a complete lattice-ordered quasigroup with respect to the inclusion order and $G$ is embedded as a directed quasigroup in $G^{\#}$. This completes the proof of Theorem 2 . Obviously, the theorem remains true when each occurrence of "quasigroup" is replaced by "loop".

3. Completeness and linear order. Suppose $L$ is a compete fully ordered loop with a smallest positive element $e$. Then $L$ is generated by $e$ and so as Zelinsky has shown [9], $L$ must be the set of integers with ordinary addition and the usual order.

Henceforth we will assume that $L$ is a complete fully ordered loop with no smallest positive member. Thus if $a, b \in L$ with $a<b$, then $0<b-a$ and so there is a member $c$ of $L$ such that $0<c<b-a$. Thus $a<c+a<b$, and hence we conclude that between any two members of $L$ there is a third member of $L$.

LEMMA 4. Let $n$ be a fixed positive integer and let $L_{n}=\left\{n a \mid a \in L^{+}\right\}$. Then $L_{n}=L^{+}$(here $L^{+}$denotes the set of nonnegative members of $\left.L\right)$.

Proof. We proceed by induction on $n$. The proposition is trivial for $n=1$. Suppose that $n \geqq 2$ and that $L_{n-1}=L^{+}$. Let $z \in L^{+}$and let

$$
\begin{array}{ll}
A=\left\{a \in L^{+} \mid n a \leqq z\right\}, & A_{n}=\{n a \mid a \in A\}, \\
B=\left\{a \in L^{+} \mid n a \geqq z\right\}, & B_{n}=\{n a \mid a \in B\} .
\end{array}
$$

Suppose that $z \notin A_{n} \cup B_{n}$. We know that $A \neq \varnothing$ since $0 \in A$ and $B \neq \varnothing$ since $z \leqq n z$ so that $n z \in B$. In fact, $A \cup B=L^{+}$, and if $a \in A$ and $b \in B$ then $n a<z<n b$ so that $a<b$. Thus $A$ is bounded above and $B$ is bounded below. Further, the least upper bound of $A$ equals the greatest lower bound of $B$. Let $a_{0}$ 
be the least upper bound of $A$. We know that $a_{0} \in A \cup B$. Suppose $a_{0} \in A$ and let $u$ be the greatest lower bound of $B_{n}$. Then $n a_{0}<z \leqq u$. Using the induction hypothesis, we find $y \in L^{+}$such that $(n-1) a_{0}<(n-1) y<u-a_{0}$. Then $a_{0}<y$ and $n a_{0}<(n-1) y+a_{0}$. Also $a_{0}<-(n-1) y+u$. Now we find $x \in L^{+}$such that $a_{0}<x<-(n-1) y+u$. Thus $n a_{0}<(n-1) y+a_{0}<(n-1) y+$ $x<u$. If $x \leqq y$ then $n a_{0}<n x<u$. But since $x \in B$, this would lead to a contradiction. On the other hand, if $y<x$ then $n a_{0}<n y<u$. But since $y \in B$, this also would lead to a contradiction. Hence if $a_{0} \in A$ then $z \in L_{n}$. The argument is similar in case $a_{0} \in B$. This completes the induction argument.

Now fix $a>0$. For each positive integer $n$ there is, by Lemma 4, a unique positive element $x$ of $L$ such that $n x=a$. Henceforth $x$ will be called the nth right root of $a$, and we will write $x=(1 / n) a$.

Lemma 5. Let $b, c \in L^{+}$with $b<c$. There are positive integers $m$ and $n$ such that $b \leqq m((1 / n) a)<c$.

Proof. Suppose that $(1 / n) a \geqq-b+c \quad(n=1,2, \cdots)$, so that $a \geqq$ $n(-b+c)(n=1,2, \cdots)$. Then by Theorem 1 we may conclude that $-b+c \leqq 0$, which is a contradiction. So for some positive integer $n$, we have $(1 / n) a<-b+c$. Again by Theorem 1 , there is a positive integer $m$ such that $(m-1)((1 / n) a)<b \leqq m((1 / n) a)$. If $m((1 / n) a) \geqq c$ then $(1 / n) a=$ $-(m-1)((1 / n) a)+m((1 / n) a) \geqq-(m-1)((1 / n) a)+c>-b+c$, which is a contradiction. Thus we have $b \leqq m((1 / n) a)<c$.

We conclude from Lemma 4 and Lemma 5 that the set $D=\{m((1 / n) a) \mid m$ and $n$ are positive integers $\}$ is dense in $L^{+}$, and hence the set $D \cup$ $\{0-d \mid d \in D\}$ is a countable dense subset of $L$ which is order-isomorphic to the set of rational numbers. It is a well-known result of Cantor [4] that a (conditionally) complete fully ordered set containing a dense subset order-isomorphic to the rational numbers is in fact order-isomorphic to the real numbers. Thus we have the following result:

THEOREM 6. If $L$ is a complete fully ordered loop then either $L$ is isomorphic to the integers with ordinary addition or $L$ is a loop with the real numbers as its underlying set.

COROLLARY 7. If $G$ is a complete fully ordered quasigroup then either $G$ is isotopic to the integers with ordinary addition or $G$ has the real numbers as its underlying set.

Proof. Suppose $G$ is a complete fully ordered quasigroup and $a, b \in G$. Then $G$ with the new operation $x e y=(x-b)+(-a+y)$ is a complete fully ordered loop with neutral element $a+b$ (see [3, p. 56]). Thus the corollary follows from Theorem 6 .

COROLLARY 8. If a filly ordered loop or quasigroup is integrally closed then it can be embedded as an ordered set in the real numbers. 
The converse of Corollary 8 is not true even for groups. For if $G$ is the direct product $Z \times Z$ of two copies of the integers with the lexicographic order then $G$ is embeddable as an ordered set in the real numbers but $G$ is not integrally closed since $n(0,1)<(2,0)(n=1,2, \cdots)$ and $(0,1)>(0,0)$.

We have the following obvious characterization of complete fully ordered loops:

COROLlary 9. L is a complete fully ordered loop if and only if its addition operation can be characterized as a function $f: L \times L \rightarrow L$ where $L$ is either the set of integers or real numbers and

(i) $f(a, L)=f(L, a)=L$ for each $a \in L$,

(ii) $f$ is strictly increasing in each argument, and

(iii) there is an element $e$ of $L$ such that, for each $a \in L$,

$$
f(e, a)=f(a, e)=a \text {. }
$$

For convenience, $e$ may be chosen as 0 . If we omit condition (iii), we have a characterization of complete fully ordered quasigroups. In either case, such a function $f$ is obviously continuous. Thus we have the following generalization of an early result of Hölder [7].

COROLLARY 10. If $L$ is a complete fully ordered, strongly power associative loop then $L$ is either the additive group of integers or the additive group of real numbers.

Proof. Aczél has shown (see [1, p. 435]) that a strongly power associative loop with a continuous addition and whose underlying set is the real numbers is isomorphic to the additive group of real numbers.

For this corollary, it is not enough that we assume $L$ is power associative as the following example shows.

EXAMPLE. We define an operation $(\oplus)$ on the set $R$ of real numbers by

(i) $a \oplus b=a+b$ if $a \geqq 0$ or $b \geqq 0$, and

(ii) $a \oplus b=a+b-a b$ otherwise.

The set $R$ with this operation and the usual order is a complete fully ordered loop, however it is not associative since $(-1 \oplus-1) \oplus 1 \neq$ $-1 \oplus(-1 \oplus 1)$. Thus according to Corollary 10 , the operation $(\oplus)$ is not strongly power associative. But it is power associative since the set of positive elements and the set of negative elements are each subsemigroups of $R$.

We note that, in this example, the subloop $K$ generated by 1 is the set $Q$ of all rational numbers. This suggests the following result.

COROLlaRY 11. If $L$ is a compleie fully ordered loop and if $a$ is $a$ member of $L$ that does not generate a subgroup then the subloop $K$ generated by $a$ is dense in $L$. 
Proof. $K$ is not a subgroup, thus $K$ has no smallest positive element, and so $K$ must be dense in $L$.

\section{REFERENCES}

1. J. Aczél, Quasigroups, nets, and nomograms, Advances in Math. 1 (1965), fasc. 3 , 383-450. MR 33 \#1395.

2. G. Birkhoff, Lattice theory, 3rd ed., Amer. Math. Soc. Colloq. Publ., vol. 25, Amer. Math. Soc., Providence, R.I., 1967. MR 37 \#2638.

3. R. H. Bruck, A survey of binary systems, Ergebnisse der Mathematik und ihrer Grenzgebiete, Neue Folge, Heft 20. Reihe: Gruppentheorie, Springer-Verlag, Berlin, 1958. MR 20 \#76.

4. G. Cantor, Einfach geordnete Menge, Math. Ann. 46 (1895), 481-512.

5. T. Evans, Lattice-ordered loops and quasigroups, J. Algebra 16 (1970), 218-226. MR 41 \#8315.

6. L. Fuchs, Partially ordered algebraic systems, Pergamon Press, Oxford; AddisonWesley, Reading, Mass., 1963. MR 30 \#2090.

7. O. Hölder, Die Axiom der Quantität und die Lehre vom Mass, Ber. Verh. Sachs. Ges. Wiss. Leipzig Math. Phys. Cl. 53 (1901), 1-64.

8. K. Iseki, Structure of special ordered loops, Portugal. Math. 10 (1951), 81-83. MR 13, 313.

9. D. Zelinsky, Nonassociative valuations, Bull. Amer. Math. Soc. 54 (1948), 175-183. MR 9, 408.

Department of Mathematics, Emory University, Atlanta, Georgia 30322

Department of Mathematics, University of North Carolina at asheville, Asheville, North Carolina 28801 (Current address) 